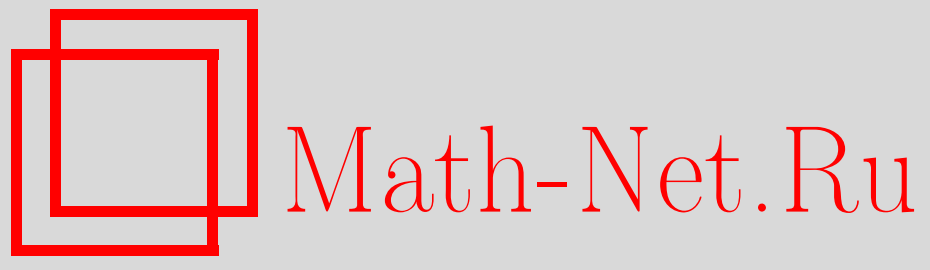

И. С. Борисов, Н. В. Володько, Асимптотические разложения распределений канонических $V$-статистик третьего порядка, Теория вероятн. и ее примен., 2015, том 60, выпуск 1, 3-24

DOI: https://doi.org/10.4213/tvp4603

Использование Общероссийского математического портала Math-Net.Ru подразумевает, что вы прочитали и согласны с пользовательским соглашением http://www . mathnet.ru/rus/agreement

Параметры загрузки:

IP : 54.162 .127 .20

26 апреля 2023 г., 18:22:57

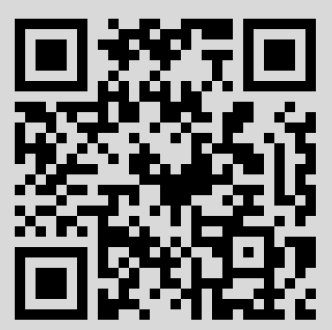


ТЕОРИЯ ВЕРОЯТНОСТЕЙ

И ЕЕ ПРИМЕНЕНИЯ

Основан в январе 1956 г.

Выходит 4 раза в год

Москва • Наука

Tом 60

Выпуск 1, 2015 год январь, февраль, март

(с) 2015 г. БОРИСОВ И. С. ${ }^{*}$, ВОЛОДЬКО Н. В.*

\section{АСИМПТОТИЧЕСКИЕ РАЗЛОЖЕНИЯ РАСПРЕДЕЛЕНИЙ КАНОНИЧЕСКИХ $V$-СТАТИСТИК ТРЕТЬЕГО ПОРЯДКА ${ }^{1)}$}

В работе получен второй член асимптотического разложения Эджворта для функций распределения канонических статистик Мизеса третьего порядка, построенных по выборкам растущего объема из последовательности независимых одинаково распределенных случайных величин. Оценка остаточного члена имеет правильный порядок малости $O\left(n^{-1}\right)$.

Ключевые слова и фразы: асимптотические разложения, канонические $U$ - и $V$-статистики, кратные ортогональные ряды.

1. Введение. В работе получены так называемые короткие асимптотические разложения Эджворта функций распределения канонических $V$-статистик третьего порядка. Метод работы основан, во-первых, на представлении канонического ядра статистики в виде специального кратного ряда (эта конструкция применялась, например, в [1] и [2]), а во-вторых, на интерпретации канонической $V$-статистики как гладкого функционала от суммы независимых случайных элементов в соответствующем банаховом пространстве и использовании уже имеющихся результатов из [3] для таких функционалов. Введем основные определения и понятия.

Пусть $X_{1}, X_{2}, \ldots$ - последовательность независимых одинаково распределенных случайных величин (с.в.), принимающих значения в произвольном сепарабельном метрическом пространстве $\mathfrak{X}$. Обозначим через

* Институт математики им. С. Л. Соболева СО РАН; Новосибирский государственный университет, Новосибирск, Россия; e-mail: sibam@math.nsc.ru, nvolodko@gmail.com

1) Работа выполнена при поддержке РФФИ (гранты № 13-01-12415, 13-01-00511 и 14-01-00220). 
$P$ распределение $X_{1}$, а через $L_{2}\left(\mathfrak{X}^{d}, P^{d}\right)$ пространство измеримых функций $f\left(t_{1}, \ldots, t_{d}\right)$, заданных на соответствующей декартовой степени пространства $\mathfrak{X}$ и имеющих конечный второй момент по продакт-мере $P^{d}$, т.е. удовлетворяющих условию $\mathbf{E} f^{2}\left(X_{1}, \ldots, X_{d}\right)<\infty$.

О п р е д е л е н и е 1 . Функция $f\left(t_{1}, \ldots, t_{d}\right) \in L_{2}\left(\mathfrak{X}^{d}, P^{d}\right)$ называется канонической (или вырожденной), если

$$
\text { E } f\left(t_{1}, \ldots, t_{k-1}, X_{k}, t_{k+1}, \ldots, t_{d}\right)=0
$$

для любого $k$ и для любых $t_{j} \in \mathfrak{X}$.

Определим каноническую статистику Мизеса формулой

$$
V_{n} \equiv V_{n}(f):=n^{-d / 2} \sum_{1 \leqslant j_{1}, \ldots, j_{d} \leqslant n} f\left(X_{j_{1}}, \ldots, X_{j_{d}}\right)
$$

где функция $f\left(t_{1}, \ldots, t_{d}\right)$ (так называемое ядро статистики) является канонической. В случае независимых наблюдений $\left\{X_{i}\right\}$ статистики такого типа (их нередко называют каноническими $V$-статистиками) изучались с середины прошлого века (обзор литературы и конкретные примеры статистик см. в [4]). Наряду с $V$-статистиками рассматриваются близкие к (2) функционалы, так называемые $U$-статистики:

$$
U_{n} \equiv U_{n}(f):=n^{-d / 2} \sum_{1 \leqslant i_{1} \neq \cdots \neq i_{d} \leqslant n} f\left(X_{i_{1}}, \ldots, X_{i_{d}}\right) .
$$

Отличие $U$-статистик от $V$-статистик состоит в отсутствии в области суммирования кратных сумм совпадающих индексов (или, как говорят, диагональных подпространств).

Метод настоящей работы основан на представлении ядра в виде специального кратного ряда. Напомним классические результаты из этой области.

Поскольку гильбертово пространство $L_{2}(\mathfrak{X}, P)$ также является сепарабельным, в нем существует счетный ортонормированный базис. Более того, положив $e_{0}(t) \equiv 1$, можно методом ортогонализации ГрамаШмидта [5] построить ортонормированный базис $\left\{e_{i}(t)\right\}_{i \geqslant 0}$, содержащий $e_{0}(t)$. Тогда $\mathbf{E} e_{i}\left(X_{1}\right) e_{j}\left(X_{1}\right)=\delta_{i j}$ для всех $i, j$ и $\mathbf{E} e_{i}\left(X_{1}\right)=0$ для всех $i \geqslant 1$ в силу условия ортогональности с $e_{0}(t)$, где $\delta_{i j}-$ символ Кронекера. Отметим, что при этом семейство функций $\left\{e_{i_{1}}\left(t_{1}\right) e_{i_{2}}\left(t_{2}\right) \cdots e_{i_{d}}\left(t_{d}\right)\right.$; $\left.i_{1}, i_{2}, \ldots, i_{d}=0,1, \ldots\right\}$ является ортонормированным базисом в пространстве $L_{2}\left(\mathfrak{X}^{d}, P^{d}\right)$ (см. [5]).

Таким образом, ядро $f\left(t_{1}, \ldots, t_{d}\right)$ рассматриваемых статистик можно разложить по базису $L_{2}\left(\mathfrak{X}^{d}, P^{d}\right)$. Если, кроме того, ядро является вырожденным, то элемент $e_{0}(t) \equiv 1$ не принимает участия в разложении, т.е. имеет место разложение

$$
f\left(t_{1}, \ldots, t_{d}\right)=\sum_{i_{1}, \ldots, i_{d}=1}^{\infty} f_{i_{1} \ldots i_{d}} e_{i_{1}}\left(t_{1}\right) \cdots e_{i_{d}}\left(t_{d}\right),
$$


при этом ряд в правой части равенства (4) сходится в норме $L_{2}\left(\mathfrak{X}^{d}, P^{d}\right)$, а само равенство понимается почти всюду относительно продакт-меры $P^{d}$.

Остановимся отдельно на случае $d=2$. Рассмотрим интегральный линейный оператор с симметричным каноническим ядром $f \in$ $L_{2}\left(\mathfrak{X}^{2}, P^{2}\right)$, переводящий $L_{2}(\mathfrak{X}, P)$ в себя. Поскольку этот линейный оператор будет вполне непрерывным и самосопряженным, то в сепарабельном гильбертовом пространстве $L_{2}(\mathfrak{X}, P)$ существует ортонормированный базис, состоящий из собственных векторов этого интегрального оператора. При этом, в силу вырожденности ядра, функция $e_{0}(t) \equiv 1$ является собственным вектором оператора, соответствующим собственному числу $\lambda_{0}=0$. Тогда для этого базиса представление (4) при $d=2$ будет выглядеть следующим образом:

$$
f\left(t_{1}, t_{2}\right)=\sum_{k=1}^{\infty} \lambda_{k} e_{k}\left(t_{1}\right) e_{k}\left(t_{2}\right)
$$

где $\lambda_{k}$ - соответствующее собственное число (см. [2], [4]). Без ограничения общности будем считать, что $\left\{\left|\lambda_{k}\right|\right\}-$ невозрастающая последовательность.

Отметим, что асимптотическому анализу распределений канонических статистик Мизеса и $U$-статистик второго порядка посвящено множество работ (см. исторический экскурс, например, в [6]-[8]). Популярность именно этого класса статистик объясняется прежде всего статистическими приложениями. Известные критерии согласия «омегаквадрат», «хи-квадрат» и некоторые другие построены с помощью статистик со структурой скалярного произведения $\left(Q S_{n}, S_{n}\right)$ в некотором гильбертовом пространстве, где $S_{n}$ - частичная сумма независимых или слабо зависимых гильбертовозначных наблюдений, $Q$ - линейный ограниченный оператор в рассматриваемом пространстве. Понятно, что $\left(Q S_{n}, S_{n}\right)$ представляет собой квадратичную форму — частный вид (с точностью до нормировки) введенных выше $V$-статистик с каноническим ядром $f\left(t_{1}, t_{2}\right)=\left(Q t_{1}, t_{2}\right)$ (при условии, что наблюдения центрированы). Отметим, что если линейный оператор $Q$ положительно определен, то указанная квадратичная форма представляет собой квадрат евклидовой нормы в соответствующем гильбертовом пространстве. Этот частный случай в настоящее время исследован достаточно полно (см. библиографию в [7] и [8]).

Далее, для произвольных $d$ при замене векторной переменной $\left(t_{1}, \ldots, t_{d}\right)$ на независимые наблюдения $\left(X_{1}, \ldots, X_{d}\right)$ частичные суммы ряда в правой части (4) сходятся к случайной величине $f\left(X_{1}, \ldots, X_{d}\right)$ в среднеквадратичном, а следовательно, и по распределению. Если при этом коэффициенты разложения $\left\{f_{i_{1} \ldots i_{d}}\right\}$ абсолютно суммируемы, то в силу теоремы Б. Леви и элементарной оценки $\mathbf{E}\left|e_{i_{1}}\left(X_{1}\right) \cdots e_{i_{d}}\left(X_{d}\right)\right| \leqslant 1$ 
ряд в (4) будет сходиться и почти наверное относительно распределения $P^{d}$ вектора $\left(X_{1}, \ldots, X_{d}\right)$. Однако при подстановке вместо аргументов ядра зависимых наблюдений только что отмеченное свойство, вообще говоря, не имеет места (см. [2]). Между тем, в выражении (2) для статистики Мизеса содержатся слагаемые вида $f\left(X_{j_{1}}, \ldots, X_{j_{d}}\right)$, где индексы аргументов могут совпадать, т.е. в этом случае аргументы не будут независимыми. Введем в рассмотрение следующее условие регулярности.

(R) ЯДро $f\left(t_{1}, \ldots, t_{d}\right)$ непрерьввно всюду на $\mathfrak{X}^{d}$ (по каждой переменной) с абсолютно суммируемьми коэффициентами $f_{i_{1} \ldots i_{d}}$ разложения (4), и все базисные элементы $e_{k}(t)$ в (4) непрерьвны и равномерно по $t$ и $k$ ограничень некоторой положительной постоянной $C_{0}$.

Отметим, что непрерывность и ограниченность базисных элементов в (4) вместе с суммируемостью коэффициентов разложения, строго говоря, не влечет непрерывность самой функции $f$, поскольку равенство в (4) изначально справедливо на множестве полной меры относительно продакт-меры $P^{d}$.

Если выполнено условие $(\mathbf{R})$, то равенство (4) превращается в тождество в $\mathfrak{X}^{d}$. Поэтому в этом тождестве вместо переменных $t_{1}, \ldots, t_{d}$ можно подставить произвольно связанные случайные величины $X_{j_{1}}, \ldots, X_{j_{d}}$ (и, в частности, наблюдения с совпадающими индексами $j_{k}$ ).

Подставив получившееся тождество для ядра в формулу (2), получим следующее представление для статистики Мизеса:

$$
V_{n}(f)=\sum_{i_{1}, \ldots, i_{d}} f_{i_{1} \ldots i_{d}} S_{n}\left(i_{1}\right) \cdots S_{n}\left(i_{d}\right)
$$

где

$$
S_{n}(k)=n^{-1 / 2} \sum_{i=1}^{n} e_{k}\left(X_{i}\right) .
$$

Иными словами, статистика Мизеса представима в виде полилинейного функционала от нормированной суммы $S_{n}=n^{-1 / 2} \sum_{i=1}^{n} \xi_{i}$ независимых одинаково распределенных бесконечномерных векторов $\xi_{i}=\left\{e_{k}\left(X_{i}\right) ; k=\right.$ $1,2, \ldots\}$. Дальнейшие рассуждения связаны с асимптотическим анализом распределений гладких функционалов от сумм независимых банаховозначных случайных величин. Эта методика идейно восходит к работам Ф. Гётце [9], [10]. Короткие асимптотические разложения, обсуждаемые в настоящей работе, содержатся в [3] (случай симметричных функционалов исследовался также в [11]). Приведем результат И. С. Борисова и Е. А. Соловьева [3] в той общности, которая нам необходима при доказательстве основного результата.

Пусть $\left\{\xi_{i} ; i \geqslant 1\right\}$ - последовательность независимых одинаково распределенных с.в. со значениями в произвольном сепарабельном банаховом пространстве $(X,\|\cdot\|)$ типа 2. Предполагается, что $\mathbf{E} \xi_{1}=0$ и 
$\mathbf{E}\left\|\xi_{1}\right\|^{4}<\infty$. Обозначим через $\left\{\tau_{i}\right\}$ независимые одинаково распределенные центрированные гауссовские с.в. в $(X,\|\cdot\|)$ с тем же ковариационным оператором, что и $\xi_{1}$. Будем предполагать, что последовательности $\left\{\xi_{i}\right\}$ и $\left\{\tau_{i}\right\}$ заданы на одном вероятностном пространстве и независимы. Кроме того, нам понадобятся следующие обозначения:

$$
\sigma^{2}=\mathbf{E}\left\|\xi_{1}\right\|^{2}, \quad \xi_{n i}=\frac{\xi_{i}}{\sigma \sqrt{n}}, \quad \tau_{n i}=\frac{\tau_{i}}{\sigma \sqrt{n}}, \quad S_{n}=\sum_{i \leqslant n} \xi_{n i}, \quad \tau=\sum_{i \leqslant n} \tau_{n i} .
$$

Далее, пусть $F(x)$ - некоторый измеримый функционал на $X$, удовлетворяющий условиям регулярности из [3]; наиболее сложно проверяемым из этих условий является следующее условие стохастической отделимости от нуля второй производной Фреше:

$$
\sup _{z} z^{-M} \mathbf{P}(D(\zeta, \eta) \leqslant z) \leqslant B
$$

для некоторого $B$ и достаточно большого $M$ (нижняя оценка для $M$ при желании может быть выписана, как, скажем, в [3]), где

$$
D(x, y)=\mathbf{E}\left(F^{(2)}(x)\left[\xi_{1}, y\right]\right)^{2}
$$

здесь и далее $\zeta$ и $\eta-$ независимые копии $\tau_{1}$. Отметим, что если функционал $F$ представляет собой квадрат евклидовой нормы в некотором сепарабельном гильбертовом пространстве, то условие (9) сводится к требованию отличия от нуля определенного числа собственных чисел ковариационного оператора. Для $V$-статистик второго порядка указанное требование касается собственных чисел $\left\{\lambda_{i}\right\}$ в $(5)$.

Далее, пусть $\psi_{t}(x)=\exp \{i t F(x)\}$. Обозначим

$$
\begin{aligned}
\varphi_{t}(x, y) & =-i t^{-1} \psi_{t}^{(3)}(x)\left[y^{3}\right] \\
= & \exp \{i t F(x)\}\left(-t^{2}\left(F^{(1)}(x)[y]\right)^{3}+3 i t F^{(1)}(x)[y] F^{(2)}(x)\left[y^{2}\right]\right. \\
& \left.\quad+F^{(3)}(x)\left[y^{3}\right]\right) \\
Q_{n}(z)= & \frac{1}{12 \pi \sigma^{3} \sqrt{n}} \int_{-\infty}^{\infty} e^{-i t z} \mathbf{E} \varphi_{t}\left(\tau, \xi_{1}\right) d t
\end{aligned}
$$

В [3] установлено, что

$$
\mathbf{P}\left(F\left(S_{n}\right) \leqslant z\right)=\mathbf{P}(F(\tau) \leqslant z)+Q_{n}(z)+R_{n}(z),
$$

где $Q_{n}(z)$ корректно определена и для любого $\nu>0$

$$
\sup _{z \in \mathbf{R}}\left|R_{n}(z)\right| \leqslant C(\nu, F, P) n^{-1+\nu} ;
$$

здесь и далее символами $C$ или $C(\cdot)$ будут обозначаться постоянные, которые не зависят от $n$, но могут зависеть от указанных в скобках 
параметров (зависимость от которых мы желаем подчеркнуть), причем постоянные одинакового написания не обязательно одни и те же в различных оценках.

3 а м е ч а н и е 1 . Если функционал $F(x)$ симметричен, т.е. $F(x)=F(-x)$, то все его производные четного (нечетного) порядка, если таковые существуют, будут также симметричными (симметричными) функционалами. В этом случае усреднение по $x$ правой части в (10а) относительно любого симметричного распределения обращается в нуль. То же самое произойдет, если усреднять указанную форму по $y$ с симметричным распределением. Иначе говоря, в этих двух случаях $Q_{n}(z) \equiv 0$.

Также отметим, что несколько более слабая версия вышеприведенного результата содержится в [3], где требовалось выполнение аналога соотношения (9) и для первой производной Фреше.

2. Основные результаты. Нашей целью является получение асимптотических разложений вида (11) с оптимальной оценкой остаточного члена, т.е. при $\nu=0$ в (12), для канонических статистик Мизеса порядка выше второго. В настоящей работе исследуется случай $d=3$. Именно в этом случае удалось проверить условие (9) стохастической отделимости от нуля второй производной Фреше функционала, имеющего вид полиномиальной формы третьего порядка от компонент суммы бесконечномерных независимых случайных векторов некоторого банахова пространства. Авторы надеются, что и в случае $d>3$ упомянутое условие также выполняется, но доказать это пока не удалось.

Будем полагать для удобства, что ядро статистики $f\left(t_{1}, t_{2}, t_{3}\right)$ и, соответственно, коэффициенты $f_{i j k}$ являются симметричными, т.е. инвариантными относительно перестановки аргументов. В противном случае можно рассматривать вместо коэффициентов $f_{i j k}$ их симметризацию

$$
\overline{f_{i j k}}=f_{i j k}+f_{i k j}+f_{j i k}+f_{j k i}+f_{k i j}+f_{k j i}
$$

в терминах которой легко переписывается соответствующая $V$-статистика.

Рассмотрим банахово пространство $l_{f}^{3}$ бесконечномерных векторов с нормой

$$
\|x\|=\left(\sum_{i} f^{(i)}\left|x_{i}\right|^{3}\right)^{1 / 3},
$$

где $f^{(i)}=\sum_{j, k}\left|f_{i j k}\right|$, и определим на нем функционал

$$
F(x)=\sum_{i, j, k} f_{i j k} x_{i} x_{j} x_{k} .
$$

Отметим, что определенный таким образом функционал $F(x)$ симметричен, т.е. $F(-x)=-F(x)$. Поэтому поправочный член $Q(z)$ в (11) будет, вообще говоря, отличным от нуля (см. замечание 1$)$. 
Мы изучаем случай независимых одинаково распределенных бесконечномерных с.в. $\xi_{i}=\left\{e_{k}\left(X_{i}\right) ; k=1,2, \ldots\right\}$, где $\left\{e_{k}(\cdot)\right\}$ - ортонормированный базис, определенный ранее. Ввиду условия $(\mathbf{R})$ можно утверждать, что $\xi_{i}$ ограничены по норме банахова пространства $l_{f}^{3}$. Так как $l_{f}^{3}$ - банахово пространство типа 2 (см. [13]), то оно содержит и введенные выше гауссовские элементы $\left\{\tau_{i}\right\}$. Так как базисные элементы в последовательности $\left\{e_{k}\left(X_{1}\right) ; k=1,2, \ldots\right\}$ удовлетворяют условию ортонормированности, то бесконечномерные векторы $\tau_{i}$ имеют независимые координаты со стандартным нормальным распределением.

Введем в рассмотрение частичную сумму $S_{n}$ независимых нормированных с.в. $\left\{\xi_{n i}\right\}$, определенных в (8). Очевидно, что в наших обозначениях $\sigma^{-3} V_{n}(f)=F\left(S_{n}\right)$. Сформулируем основной результат настоящей работы.

Теорема 1. Пусть выполнень условие $(\mathbf{R})$ и следующее предположение относительно коэффичиентов разложения в (4):

(I) для каждого фиксированного $i$ бесконечномерный вектор $\left\{f_{i i k}\right\}_{k}$ не может быть представлен в виде линейной комбинации векторов $\left\{f_{l j k}\right\}_{k}$, удовлетворяюших условию $\min (l, j)<i$.

Тогда для всех $z \in \mathbf{R}$ справедливо асимптотическое разложение

$$
\mathbf{P}\left(\sigma^{-3} V_{n}(f) \leqslant z\right)=\mathbf{P}(F(\tau) \leqslant z)+Q_{n}(z)+R_{n}(z),
$$

где $\tau$ - бесконечномерньй вектор с координатами, являюшимися независимылм стандартныли нормальныли случайными величинами, кратньй ряд $F(\tau)$ сходится почти наверное, функиия $Q_{n}(z)$ в (10b) корректно определена и

$$
\sup _{z \in \mathbf{R}}\left|R_{n}(z)\right| \leqslant C n^{-1} .
$$

3 а м е ч а н и е 2. Условие (I) линейной независимости для коэффициентов $\left\{f_{l j k}\right\}$ по сути исключает из рассмотрения ситуацию, когда ядро статистики и, стало быть, коэффициенты разложения расщепляются, т.е. представляют собой произведение функций меньшего числа переменных (в рассматриваемом случае - либо одного переменного, либо двух). В этом случае изучаемая $V$-статистика превращается в произведение двух или трех канонических $V$-статистик порядка 1 или 2. Стоит отметить, что в работе В. Бенткуса и Ф. Гётце [6] содержится асимптотический анализ распределений любых линейных комбинаций таких статистик. В частности, это открывает путь для получения соответствующих результатов для совместных распределений, а стало быть, и для элементарных преобразований наборов таких статистик, например их произведений.

3 а м е ч а н и е 3. Для каждого фиксированного вещественного $z$ положим $g_{z}(x):=\mathbf{P}(F(\tau+x)<z)$. Пусть $g_{z}^{(3)}(x)[\cdot]$ обозначает третью производную Фреше (по переменной $x$ ) функционала $g_{z}(x)$. Тогда в 
условиях теоремы 1 третья производная $g_{z}^{(3)}(x)[\cdot]$ существует и

$$
Q_{n}(z)=\frac{1}{6 \sigma^{3} \sqrt{n}} \mathbf{E} g_{z}^{(3)}(0)\left[\xi_{1}^{3}\right] .
$$

Обоснование этого равенства содержится в [3].

Ограничение $(\mathbf{R})$ в сочетании с условием (I) теоремы 1, как уже было отмечено, позволяет представлять ядро статистики в виде кратного ряда (в том числе и для совпадающих аргументов), а каноническую $V$-статистику, в свою очередь, в виде гладкого функционала от суммы бесконечномерных векторов $\left\{\xi_{n i}\right\}$. Введенные выше независимые случайные векторы и функционал, а также банахово пространство $l_{f}^{3}$ удовлетворяют необходимым условиям регулярности из [3]. Поэтому при доказательстве основного результата мы будем следовать схеме рассуждений в указанных работах.

\section{3. Стохастическая отделимость от нуля второй производ-} ной Фреше. Мы выделяем в отдельный пункт доказательство условия (9) - наиболее важного и сложно проверяемого при получении коротких асимптотических разложений. Для начала напомним, как вычисляются производные Фреше введенного выше функционала $F$ в банаховом пространстве $l_{f}^{3}$. Первая производная Фреше $F^{(1)}(x)[h]$ в точке $x$ - это линейный ограниченный функционал, а вторая производная $F^{(2)}(x)\left[h, h^{\prime}\right]$ билинейный ограниченный функционал, при этом выполнено соотношение

$$
\frac{\left|F(x+h)-F(x)-F^{(1)}(x)[h]\right|-F^{(2)}(x)[h, h] / 2}{\|h\|^{2}} \rightarrow 0, \quad\|h\| \rightarrow 0 .
$$

С учетом симметричности массива $\left\{f_{i j k}\right\}$ имеем

$$
\begin{aligned}
& F(x+h)-F(x)= \sum_{i, j, k} f_{i j k}\left(h_{i} x_{j} x_{k}+x_{i} h_{j} x_{k}+x_{i} x_{j} h_{k}+h_{i} h_{j} x_{k}\right. \\
&\left.+h_{i} x_{j} h_{k}+x_{i} h_{j} h_{k}+h_{i} h_{j} h_{k}\right) \\
&=3 \sum_{i, j, k} f_{i j k} h_{i} x_{j} x_{k}+3 \sum_{i, j, k} f_{i j k} h_{i} h_{j} x_{k} \\
&+\sum_{i, j, k} f_{i j k} h_{i} h_{j} h_{k} .
\end{aligned}
$$

Последнюю сумму в правой части (14) оценим с помощью неравенства Гёльдера для интеграла от произведения трех функций по конечной атомарной мере с носителем на целочисленной решетке $\mathbf{Z}_{+}^{3}$ и массами соответствующих атомов $\left\{\left|f_{i j k}\right|\right\}$. Тогда, принимая во внимание структуру нормы (13) банахова пространства $l_{f}^{3}$, получаем

$$
\left|\sum_{i, j, k} f_{i j k} h_{i} h_{j} h_{k}\right| \leqslant\|h\|^{3} .
$$


Таким образом, из (14) следует, что

$$
F^{(1)}(x)[h]=3 \sum_{i, j, k} f_{i j k} h_{i} x_{j} x_{k}, \quad F^{(2)}(x)\left[h, h^{\prime}\right]=6 \sum_{i, j, k} f_{i j k} x_{i} h_{j} h_{k}^{\prime} .
$$

Как и при выводе (15), легко убеждаемся в справедливости оценок

$$
\left\|F^{(1)}(x)\right\|^{*} \leqslant 3\|x\|^{2}, \quad\left\|F^{(2)}(x)-F^{(2)}(y)\right\|^{*} \leqslant 6\|x-y\|,
$$

где $\|\cdot\|^{*}$ обозначает соответствующую операторную норму. Таким образом, условия регулярности из [3] выполнены.

Лемма 1. В условиях теоремь 1 соотношение (9) выполнено для сколь угодно большого $M$.

Д о к а з а т е л ь с т в о. В силу ортонормированности последовательности $\left\{e_{i}\left(X_{1}\right)\right\}$ имеем

$$
\begin{aligned}
D(x, y) & =\mathbf{E}\left(F^{(2)}(x)\left[\xi_{1}, y\right]\right)^{2}=\mathbf{E}\left(\sum_{i, j, k} f_{i j k} x_{i} e_{j}\left(X_{1}\right) y_{k}\right)^{2} \\
& =\sum_{i}\left(\sum_{j, k} f_{i j k} x_{j} y_{k}\right)^{2} .
\end{aligned}
$$

В рамках доказательства этой леммы координаты бесконечномерных случайных векторов $\tau$ и $\eta$ будем обозначать теми же символами, но снабженными нижними индексами, что не должно привести к недоразумению с предыдущими обозначениями. Легко видеть, что в нашем случае соотношение (9) будет следовать из оценки

$$
\mathbf{P}\left(\sum_{i=1}^{m}\left(\sum_{j, k=1}^{\infty} f_{i j k} \tau_{j} \eta_{k}\right)^{2}<\varepsilon^{2}\right) \leqslant C(m) \varepsilon^{m \gamma}
$$

для сколь угодно большого $m$, где положительная постоянная $\gamma$ не зависит от $m$ и $\varepsilon$.

Введем обозначение

$$
A_{i}(j)=\sum_{k=1}^{\infty} f_{i j k} \eta_{k}
$$

Очевидно, что последовательность $\left\{\tau_{k}\right\}$ не зависит от $\left\{A_{i}(j)\right\}$. Рассмотрим условную вероятность $\mathbf{P}_{\eta}$ интересующего нас события в (16) при фиксированных $A_{i}(j)$ (или, что то же, при фиксированном векторе $\eta$ ); для нее справедлива оценка

$$
\mathbf{P}_{\eta}\left(\sum_{i=1}^{m}\left(\sum_{j=1}^{\infty} A_{i}(j) \tau_{j}\right)^{2}<\varepsilon^{2}\right) \leqslant \mathbf{P}_{\eta}\left(\bigcap_{i=1}^{m}\left\{\left|\sum_{j=1}^{\infty} A_{i}(j) \tau_{j}\right|<\varepsilon\right\}\right) \text { п.н. }
$$


Введем в рассмотрение матрицу $A=\left\{A_{i}(j)\right\}$ размера $m \times \infty$. В силу условия (I) теоремы 1 строки матрицы $A$ являются линейно независимыми с вероятностью 1 . Очевидно, что при фиксированных $\left\{\eta_{k}\right\}$ с.в. $\sum_{j=1}^{\infty} A_{i}(j) \tau_{j}, i=1,2, \ldots$, имеют совместное гауссовское распределение с ковариационной матрицей $A A^{T}$. Отсюда следует, что

$$
\mathbf{P}_{\eta}\left(\bigcap_{i=1}^{m}\left\{\left|\sum_{k=1}^{\infty} A_{i}(k) \tau_{k}\right|<\varepsilon\right\}\right) \leqslant \frac{\varepsilon^{m}}{(2 \pi)^{m / 2} \sqrt{\operatorname{det} A A^{T}}} .
$$

Далее, используя формулу полной вероятности и (17), получаем

$$
\begin{aligned}
& \mathbf{P}\left(\bigcap_{i=1}^{m}\left\{\left|\sum_{k=1}^{\infty} A_{i}(k) \tau_{k}\right|<\varepsilon\right\}\right) \\
& \leqslant \mathbf{P}\left(\bigcap_{i=1}^{m}\left\{\left|\sum_{k=1}^{\infty} A_{i}(k) \tau_{k}\right|<\varepsilon\right\} \cap\left\{\sqrt{\operatorname{det} A A^{T}}>\varepsilon^{m / 2}\right\}\right) \\
&+\mathbf{P}\left(\sqrt{\operatorname{det} A A^{T}} \leqslant \varepsilon^{m / 2}\right) \\
&= \mathbf{E}\left(\mathbf{P}_{\eta}\left(\bigcap_{i=1}^{m}\left\{\left|\sum_{k=1}^{\infty} A_{i}(k) \tau_{k}\right|<\varepsilon\right\}\right) I\left(\sqrt{\operatorname{det} A A^{T}}>\varepsilon^{m / 2}\right)\right) \\
&+\mathbf{P}\left(\sqrt{\operatorname{det} A A^{T}} \leqslant \varepsilon^{m / 2}\right) \\
& \leqslant C(m) \varepsilon^{m / 2}+\mathbf{P}\left(\sqrt{\operatorname{det} A A^{T}} \leqslant \varepsilon^{m / 2}\right),
\end{aligned}
$$

где $I(\cdot)$ — индикатор события. Наконец, в [14] доказано, что

$$
\mathbf{P}\left(\sqrt{\operatorname{det} A A^{T}} \leqslant \delta\right) \leqslant C(m) \delta \max \left(|\ln \delta|^{m-1}, 1\right) .
$$

Отсюда следует справедливость (16), а значит, и утверждение леммы.

3 а м е ч а н и е 4. Вывод оценки (18) существенно опирается на гауссовость распределения каждого элемента $A_{i}(j)$. Если порядок $V$ статистик будет больше трех, то $A_{i}(j)$ представляют собой полиномиальные формы от одной последовательности независимых стандартных нормальных случайных величин, и вопрос описания приемлемых условий на коэффициенты разложения в (4) при получении аналогов оценки (18) пока остается открытым.

4. Вспомогательные утверждения. Мы сохраним преемственность обозначений из [3], несмотря на то, что в [3] исследовался более общий случай, когда суммируемые случайные величины не обязательно одинаково распределены. В дальнейшем символом $N$ (с индексами или без) будут обозначаться те или иные непустые конечные подмножества натурального ряда. В нижеследующих утверждениях по умолчанию будет предполагаться, что минимальное число элементов в исходном $N$ таково, все элементы его разбиения в формулировках соответствующих утверждений непусты. 
Введем следующие обозначения:

$$
\begin{gathered}
S_{n k}=\sum_{i<k} \xi_{n i}+\sum_{i>k} \tau_{n i}, \\
S_{n k}(N)=\sum_{i<k, i \notin N} \xi_{n i}+\sum_{i>k, i \notin N} \tau_{n i}, \\
\delta_{n k}(N)=\sum_{i<k, i \in N} \xi_{n i}+\sum_{i>k, i \in N} \tau_{n i}, \\
\Delta_{n}(N)=\left(\frac{\operatorname{card} N}{n}\right)^{1 / 2}, \quad K=3 C_{0}^{3} \sum_{i, j, k}\left|f_{i j k}\right|,
\end{gathered}
$$

где $C_{0}$ - константа из условия $(\mathbf{R})$.

Лемма 2. Пусть F и нальи порядков 3 и $k_{0}$ соответственно, заданнье на пространстве $\mathfrak{X}^{3}$. Здесь $k_{0}$ - некоторое натуральное число. Тогда у этих функиионалов существуют все производнье Фреше, причем $F^{(3)}(x)$ и $H^{\left(k_{0}\right)}(x)$ не зависят от аргумента $x$ u

$$
\begin{aligned}
& \left\|F^{(j)}(x)\right\|^{*} \leqslant C\|x\|^{3-j}, \quad 1 \leqslant j \leqslant 3 \\
& \left\|H^{(l)}(x)\right\|^{*} \leqslant C\|x\|^{k_{0}-l}, \quad 1 \leqslant l \leqslant k_{0} .
\end{aligned}
$$

Пусть, кроме того, $\left\{N_{j} ; j \leqslant k_{0}+1\right\}$ - произвольное разбиение $N$. Тогда если

$$
\frac{|t|}{\sqrt{n}} \Delta_{n}(N)^{2-a} \leqslant \frac{1}{8 K C},
$$

mo

$$
\begin{aligned}
& \mid \mathbf{E} H\left(S_{n k}\right) \exp \left\{i t F\left(S_{n k}\right)\right\} \mid \\
& \leqslant C(\cdot)\left\{\Delta_{n}(N)^{R}+\max _{j \leqslant k_{0}+1} \mathbf{E}^{1 /(4 q)} \exp \left\{-\frac{q t^{2}}{3} \mathbf{E}_{\hat{\delta}_{n k}\left(N_{j}^{(2)}\right), \hat{\delta}_{n k}\left(N_{j}^{(3)}\right)}\right.\right. \\
&\left.\left.\times\left(F^{(3)}(\cdot)\left[\delta_{n k}\left(N_{j}^{(1)}\right), \widehat{\delta}_{n k}\left(N_{j}^{(2)}\right), \widehat{\delta}_{n k}\left(N_{j}^{(3)}\right)\right]\right)^{2}\right\}\right\}
\end{aligned}
$$

где $N_{j}^{(1)}, N_{j}^{(2)}, N_{j}^{(3)}$ - произвольное разбиение множества $N_{j}, R$ - произвольное положительное число, $\widehat{\delta}_{n k}(\cdot)$ - стандартная симметризаиия с.в. $\delta_{n k}(\cdot), q \geqslant 5 / 6$, константа $C(\cdot)$ зависит от $f$ и распределения базисных элементов.

Д о к а з а т е л ь с т в о. Лемма 2 является аналогом леммы 8 из [3] с некоторыми изменениями, необходимыми в нашем случае. Поэтому доказательство в значительной степени будет повторять соответствующие рассуждения в [3]. 
Первая группа утверждений леммы общеизвестна и приведена лишь для справки. Докажем основную часть, а именно неравенство (19). Разложим $F$ и $H$ в ряды Тейлора в точке $S_{n k}(N)$. В нашем случае эти разложения будут конечными. Имеем

$$
\begin{aligned}
& \left|\mathbf{E} H\left(S_{n k}\right) \exp \left\{i t F\left(S_{n k}\right)\right\}\right| \\
& =\mid \mathbf{E} \sum_{l=0}^{3} \frac{1}{l !} H^{(l)}\left(S_{n k}(N)\right)\left[\delta_{n k}(N)^{l}\right] \\
& \quad \times \exp \left\{i t \sum_{j=0}^{3} \frac{1}{j !} F^{(j)}\left(S_{n k}(N)\right)\left[\delta_{n k}(N)^{j}\right]\right\} \mid \\
& \leqslant \sum_{l=0}^{k_{0}} \mathbf{E} \mid \mathbf{E}_{S_{n k}(N)} \frac{1}{l !} H^{(l)}\left(S_{n k}(N)\right)\left[\delta_{n k}(N)^{l}\right] \\
& \quad \times \exp \left\{i t \sum_{j=1}^{3} \frac{1}{j !} F^{(j)}\left(S_{n k}(N)\right)\left[\delta_{n k}(N)^{j}\right]\right\} \mid .
\end{aligned}
$$

Заметим, что в правой части (20) содержатся члены вида

$$
J \equiv\left|\mathbf{E}_{S_{n k}(N)} L_{l}\left(\delta_{n k}(N)^{l}\right) \exp \left\{i t \widetilde{L}_{3}\left(\delta_{n k}(N)^{3}\right)\right\}\right|
$$

где $l \leqslant k_{0}, \widetilde{L}_{3}(x, x, x)=F^{(1)}(\cdot)[x]+(1 / 2) F^{(2)}(\cdot)[x, x]+(1 / 6) F^{(3)}(\cdot)[x, x, x]$, a $L_{l}\left(x^{l}\right)$ есть $l$-линейный функционал. Далее, разбиваем $N$ на $l+1$ непересекающихся подмножеств $N_{j}$. Тогда

$$
\begin{aligned}
& J \leqslant \sum_{i_{1}, \ldots, i_{l}=1}^{l+1} \mid \mathbf{E}_{S_{n k}(N)} L_{l}\left(\delta_{n k}\left(N_{i_{1}}\right), \ldots, \delta_{n k}\left(N_{i_{l}}\right)\right) \\
& \times \exp \left\{i t \widetilde{L}_{3}\left(\sum_{j \leqslant l+1, j \neq j^{*}} \delta_{n k}\left(N_{j}\right)\right)^{3}\right\} \\
& \times \mathbf{E}_{\Sigma} \exp \left\{i t \left(F^{(1)}\left(S_{n k}(N)\right)\left[\delta_{n k}\left(N_{j^{*}}\right)\right]\right.\right. \\
& +\frac{1}{2} F^{(2)}\left(S_{n k}(N)\right)\left[\delta_{n k}\left(N_{j^{*}}\right), \delta_{n k}\left(N_{j^{*}}\right)\right] \\
& +F^{(2)}\left(S_{n k}(N)\right)\left[\sum_{j \leqslant l+1, j \neq j^{*}} \delta_{n k}\left(N_{j}\right), \delta_{n k}\left(N_{j^{*}}\right)\right] \\
& +\frac{1}{6} F^{(3)}\left(S_{n k}(N)\right)\left[\delta_{n k}\left(N_{j^{*}}\right), \delta_{n k}\left(N_{j^{*}}\right), \delta_{n k}\left(N_{j^{*}}\right)\right] \\
& +\frac{1}{2} F^{(3)}\left(S_{n k}(N)\right) \\
& \times\left[\sum_{j \leqslant l+1, j \neq j^{*}} \delta_{n k}\left(N_{j}\right), \sum_{j \leqslant l+1, j \neq j^{*}} \delta_{n k}\left(N_{j}\right), \delta_{n k}\left(N_{j^{*}}\right)\right]
\end{aligned}
$$




$$
\begin{aligned}
&+ \frac{1}{2} F^{(3)}\left(S_{n k}(N)\right) \\
& \times\left.\left.\left.\times \sum_{j \leqslant l+1, j \neq j^{*}} \delta_{n k}\left(N_{j}\right), \delta_{n k}\left(N_{j^{*}}\right), \delta_{n k}\left(N_{j^{*}}\right)\right]\right)\right\} \mid \\
& \leqslant \sum_{i_{1}, \ldots, i_{l}=1}^{l+1} \mathbf{E}_{S_{n k}(N)}\left|L_{l}\left(\delta_{n k}\left(N_{i_{1}}\right), \ldots, \delta_{n k}\left(N_{i_{l}}\right)\right)\right| \\
& \times\left|\mathbf{E}_{\Sigma} \exp \left\{i t \widetilde{F}\left(\sum_{j \leqslant l+1, j \neq j^{*}} \delta_{n k}\left(N_{j}\right), \delta_{n k}\left(N_{j^{*}}\right)\right)\right\}\right|,
\end{aligned}
$$

где в каждом слагаемом $j^{*}$ не совпадает ни с одним из $i_{1}, \ldots, i_{l}$, символ $\Sigma$ обозначает семейство случайных элементов

$$
\left\{\sum_{j \leqslant l+1, j \neq j^{*}} \delta_{n k}\left(N_{j}\right), S_{n k}(N)\right\},
$$

а выражение $\exp \left\{i t \widetilde{F}\left(\sum_{j \leqslant l+1, j \neq j^{*}} \delta_{n k}\left(N_{j}\right), \delta_{n k}\left(N_{j^{*}}\right)\right)\right\}$ совпадает с выражением под знаком $\mathbf{E}_{\Sigma}$ в $(21 \mathrm{a})$. Далее, множество $N_{j^{*}}$ разобьем на три попарно непересекающихся подмножества $N_{j^{*}}^{(1)}, N_{j^{*}}^{(2)}, N_{j^{*}}^{(3)}$. Обозначим для упрощения записи

$$
\delta_{i}=\delta_{n k}\left(N_{j^{*}}^{(i)}\right), \quad i=1,2,3 .
$$

Далее мы по сути воспроизводим технический прием Ф. Гётце [9], [10], связанный с оценкой характеристических функций полилинейных функционалов (и их производных) от сумм независимых случайных элементов:

$$
\begin{aligned}
\left|\mathbf{E}_{\Sigma} \exp \left\{i t \tilde{F}\left(\sum_{j \leqslant l+1, j \neq j^{*}} \delta_{n k}\left(N_{j}\right), \delta_{n k}\left(N_{j^{*}}\right)\right)\right\}\right| \\
\leqslant\left|\mathbf{E}_{\Sigma} \exp \left\{i t\left(F^{(1)}(\cdot)\left[\delta_{1}\right]+F^{(1)}(\cdot)\left[\delta_{2}\right]+F^{(1)}(\cdot)\left[\delta_{3}\right]+\cdots\right)\right\}\right| \\
\leqslant \mathbf{E}_{\Sigma}^{1 / 2}\left|\mathbf{E}_{\delta_{1}, \delta_{2}, \Sigma} \exp \{i t(\ldots)\}\right|^{2} \\
=\mathbf{E}_{\Sigma}^{1 / 2} \exp \left\{i t \left(F^{(1)}(\cdot)\left[\widehat{\delta_{3}}\right]+F^{(2)}(\cdot)\left[\delta_{1}, \widehat{\delta_{3}}\right]+F^{(2)}(\cdot)\left[\delta_{2}, \widehat{\delta_{3}}\right]\right.\right. \\
+F^{(2)}(\cdot)\left[\sum_{j \leqslant l+1, j \neq j^{*}} \delta_{n k}\left(N_{j}\right), \widehat{\delta_{3}}\right] \\
+\frac{1}{2} F^{(2)}(\cdot)\left[\delta_{3}, \delta_{3}\right]-\frac{1}{2} F^{(2)}(\cdot)\left[\widetilde{\delta_{3}}, \widetilde{\delta_{3}}\right]+\frac{1}{2} F^{(3)}(\cdot)\left[\delta_{1}, \delta_{1}, \widehat{\delta_{3}}\right] \\
+\frac{1}{2} F^{(3)}(\cdot)\left[\delta_{2}, \delta_{2}, \widehat{\delta}_{3}\right]+\frac{1}{2} F^{(3)}(\cdot)\left[\delta_{1}, \delta_{3}, \delta_{3}\right]-\frac{1}{2} F^{(3)}(\cdot)\left[\delta_{1}, \widetilde{\delta}_{3}, \widetilde{\delta}_{3}\right] \\
+\frac{1}{2} F^{(3)}(\cdot)\left[\delta_{2}, \delta_{3}, \delta_{3}\right]-\frac{1}{2} F^{(3)}(\cdot)\left[\delta_{2}, \widetilde{\delta_{3}}, \widetilde{\delta}_{3}\right]+F^{(3)}(\cdot)\left[\delta_{1}, \delta_{2}, \widehat{\delta_{3}}\right]
\end{aligned}
$$




$$
\begin{aligned}
& +\frac{1}{2} F^{(3)}(\cdot)\left[\sum_{j \leqslant l+1, j \neq j^{*}} \delta_{n k}\left(N_{j}\right), \sum_{j \leqslant l+1, j \neq j^{*}} \delta_{n k}\left(N_{j}\right), \widehat{\delta_{3}}\right] \\
& +F^{(3)}(\cdot)\left[\sum_{j \leqslant l+1, j \neq j^{*}} \delta_{n k}\left(N_{j}\right), \delta_{1}, \widehat{\delta_{3}}\right] \\
& +F^{(3)}(\cdot)\left[\sum_{j \leqslant l+1, j \neq j^{*}} \delta_{n k}\left(N_{j}\right), \delta_{2}, \widehat{\delta_{3}}\right] \\
& +\frac{1}{2} F^{(3)}(\cdot)\left[\sum_{j \leqslant l+1, j \neq j^{*}} \delta_{n k}\left(N_{j}\right), \delta_{3}, \delta_{3}\right] \\
& \left.\left.-\frac{1}{2} F^{(3)}(\cdot)\left[\sum_{j \leqslant l+1, j \neq j^{*}} \delta_{n k}\left(N_{j}\right), \widetilde{\delta}_{3}, \widetilde{\delta}_{3}\right]\right)\right\} \\
& \leqslant \mathbf{E}_{\Sigma}^{1 / 4}\left|\mathbf{E}_{\delta_{1}, \delta_{3}, \tilde{\delta_{3}, \Sigma}} \exp \{i t(\cdots)\}\right|^{2} \\
& =\mathbf{E}_{\Sigma}^{1 / 4} \exp \left\{i t \left(F^{(2)}(\cdot)\left[\widehat{\delta_{2}}, \widehat{\delta_{3}}\right]+\frac{1}{2} F^{(3)}(\cdot)\left[\delta_{2}, \delta_{2}, \widehat{\delta_{3}}\right]\right.\right. \\
& -\frac{1}{2} F^{(3)}(\cdot)\left[\widetilde{\delta_{2}}, \widetilde{\delta_{2}}, \widehat{\delta_{3}}\right]+\frac{1}{2} F^{(3)}(\cdot)\left[\widehat{\delta_{2}}, \delta_{3}, \delta_{3}\right] \\
& -\frac{1}{2} F^{(3)}(\cdot)\left[\widehat{\delta_{2}}, \widetilde{\delta}_{3}, \widetilde{\delta}_{3}\right]+F^{(3)}(\cdot)\left[\delta_{1}, \widehat{\delta_{2}}, \widehat{\delta_{3}}\right] \\
& \left.\left.+F^{(3)}(\cdot)\left[\sum_{j \leqslant l+1, j \neq j^{*}} \delta_{n k}\left(N_{j}\right), \widehat{\delta_{2}}, \widehat{\delta_{3}}\right]\right)\right\} \\
& =: \mathbf{E}_{\Sigma}^{1 / 4} \exp \left\{i t \widetilde{F}_{1}\left(\sum_{j \leqslant l+1, j \neq j^{*}} \delta_{n k}\left(N_{j}\right), \delta_{1}, \delta_{2}, \widetilde{\delta}_{2}, \delta_{3}, \widetilde{\delta}_{3}\right)\right\} \text {. }
\end{aligned}
$$

В приведенных выкладках $\widehat{\delta}_{j}=\delta_{j}-\widetilde{\delta}_{j}$ - стандартная симметризация, т.е. здесь $\widetilde{\delta_{j}}$ и $\delta_{j}$ одинаково распределены и независимы, $j=2,3$. Отсюда следует, что

$$
\begin{aligned}
J \leqslant & \sum_{i_{1}, \ldots, i_{l}=1}^{l+1} \mathbf{E}_{S_{n k}(N)}^{3 / 4}\left|L_{l}\left(\delta_{n k}\left(N_{i_{1}}\right), \ldots, \delta_{n k}\left(N_{i_{l}}\right)\right)\right|^{4 / 3} \\
& \times \mathbf{E}_{S_{n k}(N)}^{1 / 4} \exp \left\{i t \widetilde{F}_{1}\left(\sum_{j \leqslant l+1, j \neq j^{*}} \delta_{n k}\left(N_{j}\right), \delta_{1}, \delta_{2}, \widetilde{\delta}_{2}, \delta_{3}, \widetilde{\delta_{3}}\right)\right\} .
\end{aligned}
$$

Далее, дополнительно фиксируя с.в. $\delta_{2}, \widetilde{\delta_{2}}, \delta_{3}, \widetilde{\delta_{3}}$ во внутреннем условном среднем в (22), получаем, что

$$
\begin{aligned}
J \leqslant \sum_{i_{1}, \ldots, i_{l}=1}^{l+1} & \mathbf{E}_{S_{n k}(N)}^{3 / 4}\left|L_{l}\left(\delta_{n k}\left(N_{i_{1}}\right), \ldots, \delta_{n k}\left(N_{i_{l}}\right)\right)\right|^{4 / 3} \\
\times & \mid \mathbf{E}_{S_{n k}(N)}^{1 / 4} \exp \left\{i t\left(F^{(2)}(\cdot)\left[\widehat{\delta_{2}}, \widehat{\delta_{3}}\right]+\cdots\right)\right\} \\
& \quad \times \mathbf{E}_{S_{n k}(N), \delta_{2}, \tilde{\delta_{2}}, \delta_{3}, \tilde{\delta_{3}}}^{1 / 4} \exp \left\{i t F^{(3)}(\cdot)\left[\delta_{1}, \widehat{\delta_{2}}, \widehat{\delta_{3}}\right]\right\} \mid
\end{aligned}
$$




$$
\begin{aligned}
\leqslant \sum_{i_{1}, \ldots, i_{l}=1}^{l+1} & \mathbf{E}_{S_{n k}(N)}^{3 / 4}\left|L_{l}\left(\delta_{n k}\left(N_{i_{1}}\right), \ldots, \delta_{n k}\left(N_{i_{l}}\right)\right)\right|^{4 / 3} \\
& \times\left|\mathbf{E}_{S_{n k}(N) \hat{\delta_{2}}, \hat{\delta_{3}}}^{1 / 4} \exp \left\{i t F^{(3)}(\cdot)\left[\delta_{1}, \widehat{\delta_{2}}, \widehat{\delta_{3}}\right]\right\}\right| .
\end{aligned}
$$

Теперь оценим величину

$$
\widetilde{J}:=\left|\mathbf{E}_{S_{n k}(N), \hat{\delta_{2}}, \hat{\delta_{3}}} \exp \left\{i t F^{(3)}(\cdot)\left[\delta_{1}, \widehat{\delta_{2}}, \widehat{\delta_{3}}\right]\right\}\right|
$$

на множестве элементарных исходов, задаваемом неравенствами

$$
\left\|\widehat{\delta_{2}}\right\| \leqslant \Delta_{n}(N)^{1-a_{2}}, \quad\left\|\widehat{\delta_{3}}\right\| \leqslant \Delta_{n}(N)^{1-a_{3}} .
$$

Можно воспользоваться известной оценкой

$$
\widetilde{J} \leqslant \exp \left\{-\frac{t^{2}}{3} \mathbf{E}_{S_{n k}(N), \hat{\delta_{2}, \hat{\delta_{3}}}}\left(F^{(3)}(\cdot)\left[\delta_{1}, \widehat{\delta_{2}}, \widehat{\delta_{3}}\right]\right)^{2}\right\},
$$

которая имеет место ввиду оценки

$$
\frac{2 K}{\sqrt{n}}|t|\left\|F^{(3)}(\cdot)\right\|^{*}\left\|\widehat{\delta_{2}}\right\|\left\|\widehat{\delta_{3}}\right\| \leqslant \frac{2 K C}{\sqrt{n}}|t| \Delta_{n}(N)^{2-a} \leqslant \frac{1}{4} .
$$

Далее, нетрудно видеть, что для $i=2,3$ и для любого $r \geqslant 1$

$$
\mathbf{P}\left(\left\|\widehat{\delta}_{i}\right\| \geqslant \Delta_{n}(N)^{1-a_{i}}\right) \leqslant C(r) \Delta_{n}(N)^{r a_{i}} .
$$

На основании полученных оценок продолжим оценку правой части (20):

$$
\begin{aligned}
\mid \mathbf{E} H & \left(S_{n k}\right) \exp \left\{i t F\left(S_{n k}\right)\right\} \mid \leqslant C \sum_{l=0}^{k_{0}} \mathbf{E}\left\|S_{n k}(N)\right\|^{k_{0}-l} \sum_{j=1}^{l+1} \mathbf{E}\left\|\delta_{n k}\left(N_{j}\right)\right\|^{l} \\
& \times \sum_{j^{*}=1}^{l+1} \mathbf{E}_{S_{n k}(N)}^{1 / 4} \exp \left\{-\frac{t^{2}}{3} \mathbf{E}_{S_{n k}(N) \hat{\delta_{2}}, \hat{\delta_{3}}}\left(F^{(3)}(\cdot)\left[\delta_{1}, \widehat{\delta_{2}}, \widehat{\delta_{3}}\right]\right)^{2}\right\} \\
\leqslant & C \max _{l \leqslant k_{0}} \mathbf{E}^{(q-1) / q}\left\|S_{n k}(N)\right\|^{q l /(q-1)} \\
& \times \max _{j^{*} \leqslant 4} \mathbf{E}^{1 /(4 q)} \exp \left\{-\frac{q t^{2}}{3} \mathbf{E}_{S_{n k}(N), \hat{\delta_{2}}, \hat{\delta_{3}}}\left(F^{(3)}(\cdot)\left[\delta_{1}, \widehat{\delta_{2}}, \widehat{\delta_{3}}\right]\right)^{2}\right\}
\end{aligned}
$$

Лемма 2 доказана.

Обозначим

$$
\widetilde{\Phi}_{k j}=\mathbf{E}_{\hat{\delta}_{n k}\left(N_{j}^{(2)}\right), \hat{\delta}_{n k}\left(N_{j}^{(3)}\right)}\left(F^{(3)}(\cdot)\left[\delta_{n k}\left(N_{j}^{(1)}\right), \widehat{\delta}_{n k}\left(N_{j}^{(2)}\right), \widehat{\delta}_{n k}\left(N_{j}^{(3)}\right)\right]\right)^{2} .
$$

Лемма 3. При выполнении условий

$$
\begin{aligned}
\left|t \Delta_{n}\left(N_{j}^{(1)}\right) \Delta_{n}\left(N_{j}^{(2)}\right) \Delta_{n}\left(N_{j}^{(3)}\right)\right| & \geqslant 1, \\
\frac{1}{\sqrt{n}}\left(t \Delta_{n}\left(N_{j}^{(1)}\right) \Delta_{n}\left(N_{j}^{(2)}\right)\right)^{6}\left(\Delta_{n}\left(N_{j}^{(3)}\right)\right)^{5} & <1, \\
\frac{1}{\sqrt{n}}\left(t \Delta_{n}\left(N_{j}^{(1)}\right) \Delta_{n}\left(N_{j}^{(3)}\right)\right)^{6}\left(\Delta_{n}\left(N_{j}^{(2)}\right)\right)^{5} & <1
\end{aligned}
$$


справедливо неравенство

$$
\begin{aligned}
\mathbf{E} \exp \left\{-t^{2} \widetilde{\Phi}_{k j}\right\} \leqslant & \mathbf{E}^{d /(9(d+1))} \exp \left\{-c t^{2} \widetilde{\Phi}_{k j}^{*}\left(\Gamma_{k}^{(2)}, \Gamma_{k}^{(3)}\right)\right\} \\
+ & C(\cdot)\left\{\left(n^{-1 / 2}\left(t \Delta_{n}\left(N_{j}^{(1)}\right) \Delta_{n}\left(N_{j}^{(2)}\right)\right)^{6}\left(\Delta_{n}\left(N_{j}^{(3)}\right)\right)^{5}\right)^{(d+1) / 3}\right. \\
& \left.+\left(n^{-1 / 2}\left(t \Delta_{n}\left(N_{j}^{(1)}\right) \Delta_{n}\left(N_{j}^{(3)}\right)\right)^{6}\left(\Delta_{n}\left(N_{j}^{(2)}\right)\right)^{5}\right)^{(d+1) / 3}\right\},
\end{aligned}
$$

где $d$ - произвольное положительное число, $c-$ абсолютная постоянная,

$$
\begin{gathered}
\tilde{\Phi}_{k j}^{*}(x, y)=\mathbf{E}\left(F^{(3)}(\cdot)\left[\delta_{1}^{*}, x, y\right]\right)^{2}, \\
\delta_{1}^{*}=\sum_{i \in N_{j}^{(1)}, i \neq k} \xi_{n i}, \quad \Gamma_{k}^{(l)}=\sum_{i \in N_{j}^{(l)}, i \neq k} \widehat{\tau}_{n i}, \quad l=2,3,
\end{gathered}
$$

$\widehat{\tau}_{n i}$ - симметризация гауссовских с.в. $\tau_{n i}$.

Д о к а з а т е ль с т в о. Лемма 3 является аналогом леммы 9 из работы [3], так что доказательство будет проходить по той же схеме, что и в [3]. Несложно видеть, что

$$
\widetilde{\Phi}_{k j} \equiv \mathbf{E}_{\hat{\delta_{2}}, \hat{\delta_{3}}}\left(F^{(3)}(\cdot)\left[\delta_{1}, \widehat{\delta_{2}}, \widehat{\delta_{3}}\right]\right)^{2}=\widetilde{\Phi}_{k j}^{*}\left(\widehat{\delta_{2}}, \widehat{\delta_{3}}\right)
$$

Обозначим $\Psi(x)=\mathbf{E}_{\hat{\delta_{3}}} \exp \left\{-t^{2} \widetilde{\Phi}_{k j}^{*}\left(x, \widehat{\delta_{3}}\right)\right\}$. Оценим разность $\mathbf{E} \Psi\left(\widehat{\delta_{2}}\right)-$ $\mathbf{E} \Psi\left(\Gamma_{k}^{(2)}\right)$. Для $r \leqslant k$ введем величину

$$
\widehat{\delta_{2}}(r)=\sum_{i \in N_{j}^{(2)}, i<r} \widehat{\xi}_{n i}+\sum_{i \in N_{j}^{(2)}, i>r, i \neq k} \widehat{\tau}_{n i}
$$

где $\widehat{\xi}_{n i}$ - симметризация $\xi_{n i}$. Легко видеть, что $\widehat{\delta_{2}}(0)=\Gamma_{k}^{(2)}$, а $\widehat{\delta_{2}}(k)=\widehat{\delta_{2}}$. Тогда

$$
\begin{aligned}
\left|\mathbf{E} \Psi\left(\widehat{\delta_{2}}\right)-\mathbf{E} \Psi\left(\Gamma_{k}^{(2)}\right)\right| & \leqslant \sum_{r<k, r \in N_{j}^{(2)}} \mathbf{E}\left|\Psi\left(\widehat{\delta}_{2}(r)+\widehat{\xi}_{n r}\right)-\Psi\left(\widehat{\delta}_{2}(r)+\widehat{\tau}_{n r}\right)\right| \\
= & : \sum_{r<k, r \in N_{j}^{(2)}} J_{r}
\end{aligned}
$$

Оценим $J_{r}$. Для этого воспользуемся следующим вариантом формулы Тейлора:

$$
\begin{aligned}
& \exp \left\{-t^{2} \widetilde{\Phi}_{k j}^{*}\left(x+\delta, \widehat{\delta_{3}}\right)\right\}=\exp \left\{-t^{2} \widetilde{\Phi}_{k j}^{*}\left(x, \widehat{\delta_{3}}\right)\right\} \\
& \quad \times\left\{1-t^{2}\left(\widetilde{\Phi}_{k j}^{*}\right)^{(1)}\left(x, \widehat{\delta_{3}}\right)[\delta]+\frac{t^{4}}{2}\left(\left(\widetilde{\Phi}_{k j}^{*}\right)^{(1)}\left(x, \widehat{\delta_{3}}\right)[\delta]\right)^{2}-\frac{t^{2}}{2}\left(\widetilde{\Phi}_{k j}^{*}\right)^{(2)}\left(x, \widehat{\delta_{3}}\right)\left[\delta^{2}\right]\right\} \\
& +\frac{1}{2} \int_{0}^{1}(1-\theta)^{2} \psi(x+\theta \delta, t) \exp \left\{-t^{2} \tilde{\Phi}_{k j}^{*}\left(x+\theta \delta, \widehat{\delta_{3}}\right)\right\} d \theta
\end{aligned}
$$


где

$$
\begin{aligned}
\psi(x, t)= & t^{6}\left(\left(\widetilde{\Phi}_{k j}^{*}\right)^{(1)}\left(x, \widehat{\delta_{3}}\right)[\delta]\right)^{3}-3 t^{4}\left(\widetilde{\Phi}_{k j}^{*}\right)^{(1)}\left(x, \widehat{\delta_{3}}\right)[\delta]\left(\widetilde{\Phi}_{k j}^{*}\right)^{(2)}\left(x, \widehat{\delta_{3}}\right)\left[\delta^{2}\right] \\
& +t^{2}\left(\widetilde{\Phi^{*}}{ }_{k j}\right)^{(3)}\left(x, \widehat{\delta_{3}}\right)\left[\delta^{3}\right] .
\end{aligned}
$$

Выражения $\left(\widetilde{\Phi}_{k j}^{*}\right)^{(s)}\left(x, \widehat{\delta_{3}}\right)$ здесь обозначают производные Фреше функционала $\widetilde{\Phi}_{k j}^{*}\left(x, \widehat{\delta_{3}}\right)$ по первой переменной. Оценивая $J_{r}$, мы будем применять формулу Тейлора (24) при $x=\widehat{\delta}_{2}(r)$ и $\delta=\widehat{\xi}_{n r}$ или $\delta=\widehat{\tau}_{n r}$. Заметим, что

$$
\begin{aligned}
\mathbf{E}_{\widehat{\delta_{3}}}\left(\widetilde{\Phi}_{k j}^{*}\right)^{(1)}\left(x,{\widehat{\delta_{3}}}^{\prime}\right)\left[\widehat{\xi}_{n r}\right] & =\mathbf{E}_{\widehat{\delta_{3}}}\left(\widetilde{\Phi}_{k j}^{*}\right)^{(1)}\left(x,{\widehat{\delta_{3}}}^{\prime}\right)\left[\widehat{\tau}_{n r}\right]=0 \\
\mathbf{E} L_{2}(x)\left[\widehat{\xi}_{n r}^{2}\right] & =\mathbf{E} L_{2}(x)\left[\widehat{\tau}_{n r}^{2}\right]
\end{aligned}
$$

где $L_{2}(x)$ - произвольный билинейный функционал. Кроме того, выпишем оценки производных по первой переменной $\left\|\left(\widetilde{\Phi}_{k j}^{*}\right)^{(s)}\left(x, \widehat{\delta}_{3}\right)\right\|^{*}$, учитывая выражение для $\widetilde{\Phi}_{k j}^{*}$ и конкретный вид функционала $F(x)$ :

$$
\begin{aligned}
\left\|\left(\widetilde{\Phi}_{k j}^{*}\right)^{(1)}\left(x, \widehat{\delta_{3}}\right)\right\|^{*} & \leqslant 2 \mathbf{E}_{\widehat{\delta_{3}}}\left\|F^{(3)}(\cdot)\left[\delta_{1}^{*}, x, \widehat{\delta_{3}}\right]\right\|\left\|\frac{\partial F^{(3)}(\cdot)\left[\delta_{1}^{*}, x, \widehat{\delta_{3}}\right]}{\partial x}\right\|^{*} \\
& \leqslant C \Delta_{n}\left(N_{j}^{(1)}\right)^{2}\left\|\widehat{\delta_{3}}\right\|^{2}\|x\| ; \\
\left\|\left(\widetilde{\Phi}_{k j}^{*}\right)^{(2)}\left(x, \widehat{\delta_{3}}\right)\right\|^{*} & \leqslant 2 \mathbf{E}_{\hat{\delta_{3}}}\left(\left\|\frac{\partial F^{(3)}(\cdot)\left[\delta_{1}^{*}, x, \widehat{\delta_{3}}\right]}{\partial x}\right\|^{*}\right)^{2} \\
& \leqslant C \Delta_{n}\left(N_{j}^{(1)}\right)^{2}\left\|\widehat{\delta_{3}}\right\|^{2} \\
\left(\widetilde{\Phi}_{k j}^{*}\right)^{(3)}\left(x, \widehat{\delta_{3}}\right) & \equiv 0 .
\end{aligned}
$$

Таким образом,

$$
J_{r} \leqslant J_{r 1}+J_{r 2},
$$

где введены следующие обозначения:

$$
\begin{aligned}
J_{r 1}=C \sup _{0 \leqslant \theta \leqslant 1} \mathbf{E}\{ & \left|t^{6}\right|\left\|\left(\widetilde{\Phi}_{k j}^{*}\right)^{(1)}\left(\widehat{\delta_{2}}(r)+\theta \widehat{\xi}_{n r}, \widehat{\delta_{3}}\right)\right\|^{* 3}\left\|\widehat{\xi}_{n r}\right\|^{3} \\
& +t^{4}\left\|\left(\widetilde{\Phi}_{k j}^{*}\right)^{(1)}\left(\widehat{\delta_{2}}(r)+\theta \widehat{\xi}_{n r}, \widehat{\delta}_{3}\right)\right\|^{*} \\
& \left.\times\left\|\left(\widetilde{\Phi}_{k j}^{*}\right)^{(2)}\left(\widehat{\delta_{2}}(r)+\theta \widehat{\xi}_{n r}, \widehat{\delta_{3}}\right)\right\|^{*}\left\|\widehat{\xi}_{n r}\right\|^{3}\right\} \\
& \times \exp \left\{-t^{2} \widetilde{\Phi}_{k j}^{*}\left(\widehat{\delta_{2}}(r)+\theta \widehat{\xi}_{n r}, \widehat{\delta_{3}}\right)\right\}, \\
J_{r 2}= & C \sup _{0 \leqslant \theta \leqslant 1} \mathbf{E}\left\{\left|t^{6}\right|\left\|\left(\widetilde{\Phi}_{k j}^{*}\right)^{(1)}\left(\widehat{\delta_{2}}(r)+\theta{\widehat{\tau_{n r}}}, \widehat{\delta_{3}}\right)\right\|^{* 3}\left\|\widehat{\tau}_{n r}\right\|^{3}\right. \\
& +t^{4}\left\|\left(\widetilde{\Phi}_{k j}^{*}\right)^{(1)}\left(\widehat{\delta_{2}}(r)+\theta \widehat{\tau}_{n r}, \widehat{\delta_{3}}\right)\right\|^{*} \\
& \left.\times\left\|\left(\widetilde{\Phi}_{k j}^{*}\right)^{(2)}\left(\widehat{\delta_{2}}(r)+\theta \widehat{\tau}_{n r}, \widehat{\delta_{3}}\right)\right\|^{*}\left\|\widehat{\tau}_{n r}\right\|^{3}\right\} \\
& \times \exp \left\{-t^{2} \widetilde{\Phi}_{k j}^{*}\left(\widehat{\delta}_{2}(r)+\theta \widehat{\tau}_{n r}, \widehat{\delta_{3}}\right)\right\} .
\end{aligned}
$$


Принимая во внимание неравенство $\left|t \Delta_{n}\left(N_{j}^{(1)}\right) \Delta_{n}\left(N_{j}^{(2)}\right) \Delta_{n}\left(N_{j}^{(3)}\right)\right| \geqslant 1$, а также оценки (26), действуем по аналогии с доказательством леммы 9 в [3] и с помощью неравенства Гёльдера получаем

$$
\begin{aligned}
J_{r 1} \leqslant & C\left(t \Delta_{n}\left(N_{j}^{(1)}\right) \Delta_{n}\left(N_{j}^{(3)}\right)\right)^{6}\left(\Delta_{n}\left(N_{j}^{(2)}\right)\right)^{3} \\
& \times \sup _{0 \leqslant \theta \leqslant 1} \mathbf{E}\left\|\widehat{\xi}_{n r}\right\|^{3} \mathbf{E}_{\hat{\xi}_{n r}}^{1 / q} \exp \left\{-q t^{2} \widetilde{\Phi}_{k j}^{*}\left(\widehat{\delta}_{2}(r)+\theta \widehat{\xi}_{n r}, \widehat{\delta_{3}}\right)\right\}, \\
J_{r 2} \leqslant & C n^{-3 / 2}\left(t \Delta_{n}\left(N_{j}^{(1)}\right) \Delta_{n}\left(N_{j}^{(3)}\right)\right)^{6}\left(\Delta_{n}\left(N_{j}^{(2)}\right)\right)^{3} \\
& \times \sup _{0 \leqslant \theta \leqslant 1} \mathbf{E}^{1 / q} \exp \left\{-q t^{2} \widetilde{\Phi}_{k j}^{*}\left(\widehat{\delta_{2}}(r)+\theta \widehat{\tau}_{n r}, \widehat{\delta_{3}}\right)\right\} .
\end{aligned}
$$

Следовательно,

$$
\begin{aligned}
& \sum_{r<k, r \in N_{j}^{(2)}} J_{r} \leqslant C\left(t \Delta_{n}\left(N_{j}^{(1)}\right) \Delta_{n}\left(N_{j}^{(3)}\right)\right)^{6}\left(\Delta_{n}\left(N_{j}^{(2)}\right)\right)^{3} \\
& \quad \times\left(\sum_{r<k, r \in N_{j}^{(2)}} \sup _{0 \leqslant \theta \leqslant 1} \mathbf{E}\left\|\widehat{\xi}_{n r}\right\|^{3} \mathbf{E}_{\hat{\xi}_{n r}}^{1 / q} \exp \left\{-q t^{2} \widetilde{\Phi}_{k j}^{*}\left(\widehat{\delta_{2}}(r)+\theta \widehat{\xi}_{n r}, \widehat{\delta_{3}}\right)\right\}\right. \\
& \left.\quad+n^{-3 / 2} \sum_{r<k, r \in N_{j}^{(2)}} \sup _{0 \leqslant \theta \leqslant 1} \mathbf{E}^{1 / q} \exp \left\{-q t^{2} \widetilde{\Phi}_{k j}^{*}\left(\widehat{\delta_{2}}(r)+\theta \widehat{\tau}_{n r}, \widehat{\delta_{3}}\right)\right\}\right) .
\end{aligned}
$$

Таким образом,

$$
\begin{aligned}
& \mathbf{E} \exp \left\{-t^{2} \widetilde{\Phi}_{k j}^{*}\left(\widehat{\delta_{2}}, \widehat{\delta_{3}}\right)\right\} \\
& \leqslant C(\cdot)\left\{\mathbf{E}^{1 / q} \exp \left\{-t^{2} \widetilde{\Phi}_{k j}^{*}\left(\Gamma_{k}^{(2)}, \widehat{\delta}_{3}\right)\right\}+\left(t \Delta_{n}\left(N_{j}^{(1)}\right) \Delta_{n}\left(N_{j}^{(3)}\right)\right)^{6}\left(\Delta_{n}\left(N_{j}^{(2)}\right)\right)^{3}\right. \\
& \times\left\{\sum_{r<k, r \in N_{j}^{(2)}} \sup _{0 \leqslant \theta \leqslant 1} \mathbf{E}\left\|\widehat{\xi}_{n r}\right\|^{3} \mathbf{E}_{\hat{\xi}_{n r}}^{1 / q} \exp \left\{-t^{2} \widetilde{\Phi}_{k j}^{*}\left(\widehat{\delta}_{2}(r)+\theta \widehat{\xi}_{n r}, \widehat{\delta}_{3}\right)\right\}\right. \\
&\left.+n^{-3 / 2} \sum_{r<k, r \in N_{j}^{(2)}} \sup _{0 \leqslant \theta \leqslant 1} \mathbf{E}^{1 / q} \exp \left\{-t^{2} \widetilde{\Phi}_{k j}^{*}\left(\widehat{\delta}_{2}(r)+\theta \widehat{\tau}_{n r}, \widehat{\delta_{3}}\right)\right\}\right\} .
\end{aligned}
$$

Так как $\widehat{\delta_{2}}(r)$ и $\widehat{\delta_{2}}(r)+\theta \widehat{\tau}_{n r}$ при фиксированном $\theta$ состоят из независимых с.в., неравенство (28) по существу рекуррентное. Применяя это рекуррентное неравенство $d$ раз, получаем

$$
\begin{aligned}
\mathbf{E} \exp \left\{-t^{2} \widetilde{\Phi}_{k j}^{*}\left(\widehat{\delta_{2}}, \widehat{\delta_{3}}\right)\right\} \leqslant C(\cdot)\left\{\mathbf{E}^{1 / q^{d+1}} \exp \left\{-t^{2} \widetilde{\Phi}_{k j}^{*}\left(\Gamma_{k}^{(2)}, \widehat{\delta_{3}}\right)\right\}\right. \\
\left.\quad+\left(n^{-1 / 2}\left(t \Delta_{n}\left(N_{j}^{(1)}\right) \Delta_{n}\left(N_{j}^{(3)}\right)\right)^{6}\left(\Delta_{n}\left(N_{j}^{(2)}\right)\right)^{5}\right)^{s(d)}\right\}, \quad s(d)=\sum_{l=0}^{d} q^{-l} .
\end{aligned}
$$

Выбираем $q=1+d^{-1}$. Затем повторим приведенные выше рассуждения с заменой момента $\mathbf{E} \exp \left\{-t^{2} \widetilde{\Phi}_{k j}^{*}\left(\Gamma_{k}^{(2)}, \widehat{\delta_{3}}\right)\right\}$ на момент $\mathbf{E} \exp \left\{-t^{2} \widetilde{\Phi}_{k j}^{*}\left(\Gamma_{k}^{(2)}\right.\right.$, $\left.\left.\Gamma_{k}^{(3)}\right)\right\}$, что и закончит доказательство леммы 3. 
Следствие 1. В условиях лемм 2 и 3 при вылолнении (9) имеет место оченка

$$
\begin{aligned}
& \mathbf{E} \exp \left\{-t^{2} \widetilde{\Phi}_{k j}\right\} \leqslant C(\cdot)\left\{\left|t \Delta_{n}\left(N_{j}^{(1)}\right) \Delta_{n}\left(N_{j}^{(2)}\right) \Delta_{n}\left(N_{j}^{(3)}\right)\right|^{-2 M}\right. \\
& \quad+\left(n^{-1 / 2}\left(t \Delta_{n}\left(N_{j}^{(1)}\right) \Delta_{n}\left(N_{j}^{(2)}\right)\right)^{6}\left(\Delta_{n}\left(N_{j}^{(3)}\right)\right)^{5}\right)^{(d+1) / 3} \\
& \left.\quad+\left(n^{-1 / 2}\left(t \Delta_{n}\left(N_{j}^{(1)}\right) \Delta_{n}\left(N_{j}^{(3)}\right)\right)^{6}\left(\Delta_{n}\left(N_{j}^{(2)}\right)\right)^{5}\right)^{(d+1) / 3}+\Delta_{n}(N)^{R}\right\} .
\end{aligned}
$$

Д о к а з а т е ль с т в о. Заметим, что величина $\widetilde{\Phi}_{k j}^{*}\left(\Gamma_{k}^{(2)}, \Gamma_{k}^{(3)}\right)$ имеет такое же распределение, как величина

$$
D(\zeta, \eta)\left(\Delta_{n}\left(N_{j}^{(1)} \backslash\{k\}\right) \Delta_{n}\left(N_{j}^{(2)} \backslash\{k\}\right) \Delta_{n}\left(N_{j}^{(3)} \backslash\{k\}\right)\right)^{2} .
$$

Используя (9), получаем

$$
\begin{aligned}
\mathbf{E} \exp \left\{-t^{2} D(\zeta, \eta)\right\} & =t^{2} \int_{0}^{\infty} \mathbf{P}(D(\zeta, \eta)<x) \exp \left\{-t^{2} x\right\} d x \\
& \leqslant e^{-t^{2}}+B t^{2} \int_{0}^{1} x^{M} \exp \left\{-t^{2} x\right\} d x \leqslant C(B)|t|^{-2 M}
\end{aligned}
$$

откуда следует, что

$\mathbf{E} \exp \left\{-t^{2}\left(\widetilde{\Phi}_{k j}^{*}\left(\Gamma_{k}^{(2)}, \Gamma_{k}^{(3)}\right)\right)^{2}\right\} \leqslant C(B)\left(|t| \Delta_{n}\left(N_{j}^{(1)}\right) \Delta_{n}\left(N_{j}^{(2)}\right) \Delta_{n}\left(N_{j}^{(3)}\right)\right)^{-2 M}$.

Лемма 4. Пусть $H$ и F удовлетворяют условиям леммь 2. Тогда для любого $t \in \mathbf{R}$ при вьполнении (9)

$$
|\mathbf{E} H(\tau) \exp \{i t F(\tau)\}| \leqslant C(\cdot)|t|^{-3} .
$$

Д о к а з а т е л с с в о содержится в работах [3] и [12].

5. Доказательство теоремы 1. Введем обозначения:

$$
\begin{gathered}
f_{n}(t)=\mathbf{E} \exp \left\{i t F\left(S_{n}\right)\right\}, \quad f(t)=\mathbf{E} \exp \{i t F(\tau)\} \\
h(t)=i t \int_{-\infty}^{\infty} e^{i t x} d Q_{n}(x) .
\end{gathered}
$$

В силу леммы 4 функция $f(t)+h(t)$ абсолютно интегрируема на прямой. Поэтому ее обратное преобразование Фурье является функцией ограниченной вариации с ограниченной производной. На основании формулы Берри (см. [15]) можно получить оценку

$$
\sup _{x \in \mathbf{R}}\left|R_{n}(x)\right| \leqslant \int_{|t| \leqslant n}\left|f_{n}(t)-f(t)-h(t)\right||t|^{-1} d t+C(F, B) n^{-1} .
$$


Разобьем область интегрирования $|t| \leqslant n$ на следующие две части:

$$
\{|t| \leqslant D\}, \quad\{D \leqslant|t| \leqslant n\},
$$

где $D \geqslant 1-$ некоторая константа. Оценим сначала интеграл по второй части. Положим $\Delta_{n}\left(N^{(i)}\right) \sim t^{-1 / 3+\alpha}, i=1,2,3, \alpha>0$. Заметим, что при выборе достаточно малого $\alpha$ будут выполнены все условия лемм 3 и 4 , наложенные на $\Delta_{n}\left(N^{(i)}\right)$. Кроме того, выберем константу $D$ так, чтобы было выполнено соотношение

$$
\Delta_{n}\left(N^{(1)}\right)^{2}+\Delta_{n}\left(N^{(2)}\right)^{2}+\Delta_{n}\left(N^{(3)}\right)^{2} \leqslant 1,
$$

необходимое в силу определения $\Delta_{n}\left(N^{(i)}\right)$. Несложно вычислить, что, например, при $\alpha \leqslant 1 / 6$ можно положить $D=27$.

Снова воспользуемся методом композиции (или, что то же, методом Линдеберга). Обозначим $\psi_{t}(x)=\exp \{i t F(x)\}$. Тогда справедлива оценка

$$
\begin{aligned}
& \left|f_{n}(t)-f(t)-h(t)\right|=\left|\mathbf{E} \psi_{t}\left(S_{n}\right)-\mathbf{E} \psi_{t}(\tau)-h(t)\right| \\
& \quad \leqslant \sum_{k \leqslant n}\left|\mathbf{E} \psi_{t}\left(S_{n k}+\xi_{n k}\right)-\mathbf{E} \psi_{t}\left(S_{n k}+\tau_{n k}\right)-i t \mathbf{E} \varphi_{t}\left(\tau, \xi_{n k}\right)\right| .
\end{aligned}
$$

Разложим функции $\psi_{t}\left(S_{n k}+\xi_{n k}\right)$ и $\psi_{t}\left(S_{n k}+\tau_{n k}\right)$ в ряд Тейлора в точке $S_{n k}$. Отметим, что в силу определения $\varphi_{t}$ мы имеем $i t \varphi_{t}(x, y)=\psi_{t}^{(3)}(x)\left[y^{3}\right]$. Следовательно,

$$
\begin{aligned}
J \equiv & \left|f_{n}(t)-f(t)-h(t)\right| \leqslant \sum_{k \leqslant n}\left|\mathbf{E} \psi_{t}^{(1)}\left(S_{n k}\right)\left[\xi_{n k}\right]-\mathbf{E} \psi_{t}^{(1)}\left(S_{n k}\right)\left[\tau_{n k}\right]\right| \\
& +\sum_{k \leqslant n} \frac{1}{2}\left|\mathbf{E} \psi_{t}^{(2)}\left(S_{n k}\right)\left[\xi_{n k}^{2}\right]-\mathbf{E} \psi_{t}^{(2)}\left(S_{n k}\right)\left[\tau_{n k}^{2}\right]\right| \\
& +\sum_{k \leqslant n} \frac{1}{6}\left|\mathbf{E} \psi_{t}^{(3)}\left(S_{n k}\right)\left[\xi_{n k}^{3}\right]-\mathbf{E} \psi_{t}^{(3)}(\tau)\left[\xi_{n k}^{3}\right]\right| \\
& +\sum_{k \leqslant n} \frac{1}{6} \int_{0}^{1}\left\{\left|\mathbf{E} \psi_{t}^{(4)}\left(S_{n k}+\theta \xi_{n k}\right)\left[\xi_{n k}^{4}\right]\right|+\left|\mathbf{E} \psi_{t}^{(4)}\left(S_{n k}+\theta \tau_{n k}\right)\left[\tau_{n k}^{4}\right]\right|\right\} d \theta \\
= & \sum_{k \leqslant n} \frac{1}{6}\left(J_{n k}^{(3)}+J_{n k}^{(4)}\right) .
\end{aligned}
$$

Символами $J_{n k}^{(3)}$ и $J_{n k}^{(4)}$ обозначаются слагаемые, содержащие соответственно третьи и четвертые производные Фреше функционала $\psi_{t}(\cdot)$. Первые две суммы в промежуточном звене (29) обращаются в нуль в силу совпадения ковариационных матриц векторов $\xi_{n k}$ и $\tau_{n k}$.

Оценим $J_{n k}^{(4)}$. Имеем

$$
\begin{aligned}
\psi_{t}^{(4)}(x)\left[h^{4}\right]= & \exp \{i t F(x)\}\left[t^{4}\left(F^{(1)}(x)[h]\right)^{4}-6 i t^{3}\left(F^{(1)}(x)[h]\right)^{2} F^{(2)}(x)\left[h^{2}\right]\right. \\
& \left.-4 t^{2} F^{(1)}(x)[h] F^{(3)}(x)\left[h^{3}\right]-3 t^{2}\left(F^{(2)}(x)\left[h^{2}\right]\right)^{2}\right] .
\end{aligned}
$$


Применяя леммы 3 и 4, получаем в области $\{D \leqslant|t| \leqslant n\}$ :

$$
J_{n k}^{(4)} \leqslant C(\cdot) n^{-2} t^{4}\left(t^{-R}+\left(n^{-1 / 2} t^{1 / 3+17 \alpha}\right)^{d}+t^{-6 \alpha M}\right),
$$

где $R$ и $d-$ произвольные положительные числа. Полагая $\alpha>0$ таким, чтобы выполнялось неравенство $1 / 3+17 \alpha<1 / 2$, и затем $M>0$ таким, чтобы $6 \alpha M$ было достаточно велико, а также выбирая достаточно большие $R$ и $d$, получаем, что

$$
\int_{D \leqslant|t| \leqslant n} t^{-1} \sum_{k \leqslant n} J_{n k}^{(4)} d t \leqslant C(\cdot) n^{-1} .
$$

Остается оценить величину

$$
J_{n k}^{(3)}=\left|\mathbf{E} \psi_{t}^{(3)}\left(S_{n k}\right)\left[\xi_{n k}^{3}\right]-\mathbf{E} \psi_{t}^{(3)}(\tau)\left[\xi_{n k}^{3}\right]\right| .
$$

Воспользуемся методом Линдеберга для оценки математического ожидания функционала $\widetilde{\psi_{t}}(\cdot)=\psi_{t}^{(3)}(\cdot)\left[\xi_{n k}^{3}\right]$. Имеем

$$
\begin{aligned}
J_{n k}^{(3)}:= & \left|\mathbf{E} \widetilde{\psi_{t}}\left(S_{n k}\right)-\mathbf{E} \widetilde{\psi_{t}}(\tau)\right| \\
\leqslant & \left|\mathbf{E} \widetilde{\psi_{t}}\left(S_{n k}\right)-\mathbf{E} \widetilde{\psi_{t}}\left(S_{n k}+\tau_{n k}\right)\right| \\
& +\sum_{l \leqslant k-1}\left|\mathbf{E} \widetilde{\psi_{t}}\left(S_{n l}+\xi_{n l}\right)-\mathbf{E} \widetilde{\psi_{t}}\left(S_{n l}+\tau_{n l}\right)\right| \\
=: & \widetilde{J}_{n k}^{(3)}+\sum_{l \leqslant k-1} \widetilde{J}_{n l}^{(3)} .
\end{aligned}
$$

Разложим $\widetilde{\psi_{t}}\left(S_{n k}+x\right)$ в ряд Тейлора в точке $S_{n k}$. Тогда для $l<k$

$$
\begin{aligned}
& \widetilde{J}_{n l}^{(3)} \leqslant \frac{1}{2} \int_{0}^{1}(1-\theta)^{2}\left(\left|\mathbf{E} \widetilde{\psi}_{t}^{(3)}\left(S_{n l}+\theta \xi_{n l}\right)\left[\xi_{n l}^{3}\right]\right|+\left|\mathbf{E} \widetilde{\psi}_{t}^{(3)}\left(S_{n l}+\theta \tau_{n l}\right)\left[\tau_{n l}^{3}\right]\right|\right) d \theta, \\
& \widehat{J}_{n k}^{(3)}=\frac{1}{2}\left|\mathbf{E} \widetilde{\psi}_{t}^{(2)}\left(S_{n l}\right)\left[\tau_{n l}^{2}\right]\right|+\frac{1}{2} \int_{0}^{1}(1-\theta)^{2}\left|\mathbf{E} \widetilde{\psi}_{t}^{(3)}\left(S_{n l}+\theta \tau_{n l}\right)\left[\tau_{n l}^{3}\right]\right| d \theta .
\end{aligned}
$$

Аналогично предыдущему, выбрав подходящие числа $R, d, \alpha$ и $M$ и используя леммы 3 и 4, можно получить следующие оценки:

$$
\widetilde{J}_{n l}^{(3)} \leqslant C(\cdot) \mathbf{E}\left|\xi_{n k}\right|^{3} \mathbf{E}\left|\xi_{n l}\right|^{3} t^{-1}=C(\cdot) n^{-3} t^{-1}, \quad \widehat{J}_{n k}^{(3)} \leqslant C(\cdot) n^{-5 / 2} t^{-1} .
$$

Отсюда следует, что

$$
J_{n k}^{(3)} \leqslant C(\cdot) n^{-2} t^{-1}, \quad \int_{D}^{n} t^{-1} \sum_{k \leqslant n} J_{n k}^{(3)} d t \leqslant C(\cdot) n^{-1} .
$$

Для оценки величины $\int_{0}^{D} t^{-1}\left|f_{n}(t)-f(t)-h(t)\right| d t$ можно повторить доказательство предыдущей части в упрощенном виде: множитель $\exp \{i t F(\cdot)\}$ всюду оценить по модулю сверху единицей. В силу 
того, что $\mathbf{E}\left\|F^{(i)}\left(S_{n l}\right)\right\|^{j}<\infty$ для любых $i$ и $j$, можно заметить, что $J_{n k}^{(3)}+J_{n k}^{(4)} \leqslant C(\cdot) n^{-2} t$, откуда следует оценка

$$
\int_{0}^{D} t^{-1} \sum_{k \leqslant n}\left(J_{n k}^{(3)}+J_{n k}^{(4)}\right) d t \leqslant C(\cdot) n^{-1} .
$$

Теорема 1 доказана.

\section{СПИСОК ЛИТЕРАТУРЫ}

1. Rubin H., Vitale R. Asymptotic distribution of symmetric statistics. - Ann. Statist., 1980, v. 8, № 1, p. 165-170.

2. Борисов И. С., Володько Н. В. Ортогональные ряды и предельные теоремы для канонических $U$ - и $V$-статистик от стационарно связанных наблюдений. - Матем. тр., 2008, v. 11, № 1, с. 25-48.

3. Борисов И.С., Соловьев Е.А. Аппроксимация второго порядка распределений гладких функционалов от сумм независимых банаховозначных случайных элементов. - Тр. Ин-та матем. СО РАН, 1993, т. 2, с. 3-31.

4. Королюк В. С., Боровских Ю. В. Теория $U$-статистик. Киев: Наукова думка, $1989,383 \mathrm{c}$.

5. Колмогоров A.H., Фомин C.B. Элементы теории функций и функционального анализа. М.: Наука, 1968, 496 с.

6. Bentkus V., Götze F. Optimal bounds in non-Gaussian limit theorems for $U$ statistics. - Ann. Probab., 1999, v. 27, № 1, p. 454-521.

7. Гётие Ф., Зайцев А.Ю. Равномерные оценки точности аппроксимации короткими асимптотическими разложениями в центральной предельной теореме для квадратичных форм. - Зап. науч. семин. ПОМИ РАН, 2010, т. 384, с. 105-153.

8. Fujikoshi Y., Ulyanov V. V., Shimizu R. Multivariate Statistics: High-Dimensional and Large-Sample Applications. Hoboken: Wiley, 2010, 533 p.

9. Götze F. On the rate of convergence in the central limit theorem in Banach spaces. Preprints in Statistics, №68. Cologne: Univ. of Cologne, 1981.

10. Götze F. On the rate of convergence in the central limit theorem in Banach spaces. Ann. Probab., 1986, v. 14, № 3, p. 922-942.

11. Виноградова Т. Р. О точности нормальной аппроксимации на множествах, задаваемых гладкой функцией. I, II. - Теория вероятн. и ее примен., 1985, т. 30, в. 2, c. $219-229$; в. 3 , с. $554-557$.

12. Борисов И. С. Аппроксимация распределений гладких функционалов от сумм независимых случайных элементов в банаховых пространствах. - Асимптотический анализ распределений случайных процессов. - Тр. Ин-та матем. СО АН CCCP, 1989, т. 13 , с. $7-40$.

13. Ledoux M., Talagrand M. Probability in Banach Spaces. Isoperimetry and Processes. Berlin: Springer-Verlag, 1991, 480 p.

14. Volodko N. V. Small deviations of determinants of random matrices with Gaussian entries. - Statist. Probab. Lett., 2014, v. 84, p. 48-53.

15. Петров В. В. Суммы независимых случайных величин. М.: Наука, 1972, 414 с.

Поступила в редакцию 24.VII.2013

Исправленный вариант 21.VII. 2014 